\title{
Actividad desarrollada por los Servicios de Medicina Interna de los hospitales públicos de Galicia
}

\author{
Activity developed by the Internal Medicine Services \\ of public hospitals in Galicia
}

\author{
Susana Rivera García, Fernando de la Iglesia Martínez \\ Servicio de Medicina Interna. Complejo Hospitalario Universitario A Coruña (CHUAC). SERGAS. A Coruña
}

\begin{abstract}
Resumen
OBJETIVO: Mostrar la actividad real que realizan los internistas en los distintos hospitales públicos gallegos. La finalidad es obtener una foto de la organización de los Servicios de Medicina Interna (SMI) de nuestra Comunidad Autónoma.
\end{abstract}

MATERIAL Y MÉTODOS: La Sociedad Gallega de Medicina Interna (SOGAMI) realizó una encuesta con diferentes aspectos sobre la actividad desarrollada por los SMI de cada hospital público de Galicia durante el año 2009. Fue contestada por los representantes-vocales de la SOGAMl de cada hospital o, en su defecto, por otro miembro del centro encuestado. Para comparar algunas variables se clasificaron los hospitales en dos niveles: Hospitales Comarcales y Hospitales no Comarcales, incluyendo éste último a los Hospitales de Área y a los Hospitales de Referencia. Se realizó un estudio descriptivo de las variables expresando las medidas de centralización y dispersión de las variables cuantitativas y el valor absoluto y porcentaje de las variables cualitativas.

RESULTADOS: Participaron catorce de los dieciséis hospitales públicos de Galicia. El 22\% de las camas de los centros hospitalarios gallegos son responsabilidad de los Servicios de Medicina Interna. La media de camas a cargo de un adjunto de Medicina Interna es de 8,5 $\pm 2,0$. Hay 253 internistas trabajando en los 14 Hospitales del estudio, de los cuales 28 trabajan en otras áreas del hospital no dependientes de Medicina Interna. La estancia media de hospitalización en el año 2009 de los SMl fue de $9,8 \pm 1,3$ días. La media de guardias de Medicina Interna por adjunto en Galicia es de 3,5 \pm 0,9 al mes. El 7,6\% de los internistas trabajan sólo en Consultas Externas. Sólo en el 42,9\% de los hospitales hay residentes en dichas consultas. La media de interconsultas hospitalarias es de 13,3 \pm 11,4 por hospital a la semana.

CONCLUSIONES: Medicina Interna es responsable de la asistencia sanitaria hospitalaria de una gran parte de la población gallega. Existe una heterogeneidad en los Servicios de Medicina Interna de nuestra Comunidad Autónoma que no es atribuible sólo a los distintos niveles de los hospitales.

PALABRAS CLAVE: Medicina Interna. Encuesta. Actividad asistencial.

\section{Introducción}

El conocimiento de la actividad hospitalaria constituye un elemento de gran trascendencia en el desarrollo de la política sanitaria global. La descripción del trabajo realizado y del perfil de los pacientes atendidos en nuestros servicios debe servir para diseñar el futuro de nuestra especialidad, trasmitir su importancia a los gestores y a otros especialistas médicos y tomar referencias en la definición de los estándares de nuestra propia práctica clínica ${ }^{1,2}$.

\section{Abstract}

OBJECTIVE: Showing the actual activity performed by internists in various public hospitals in Galicia. The aim is to get a picture of the organization of Internal Medicine Service (SMI) of our region.

MATERIALS AND METHODS: Galician Society of Internal Medicine (SOGAMI) conducted a survey on different aspects of the activity developed by SMl of each public hospital in Galicia in 2009. It was answered by the representatives-members of each hospital's SOGAMI or, alternatively, by another member of respondent centre. To compare some variables, hospitals were classified into two levels: County Hospitals and Not County Hospitals, including the latter both the Area Hospitals and Reference Hospitals. A descriptive study of variables was performed, expressing the measures of central tendency and dispersion for quantitative variables and the absolute value and percentage of qualitative variables.

RESULTS: Fourteen of the sixteen public hospitals in Galicia participated. $22 \%$ of the beds of Galician hospitals are the responsibility of Internal Medicine Services. The average number of beds by a doctor of Internal Medicine is $8.5 \pm 2.0$. There are 253 internist doctors working in the 14 hospitals in the study, 28 of them work in other areas of the hospital not dependent of Internal Medicine. The average stay in hospital in 2009, the SMI was $9.8 \pm 1.3$ days. The average guards of internal medicine by internist in Galicia are $3.5 \pm 0.9$ a month. $7.6 \%$ of internists work only in external consultations. Only in $42.9 \%$ of hospitals there are residents in those consultations. The average hospital inter-consultations are $13.3 \pm$ 11.4 per hospital per week.

CONCLUSIONS: Internal Medicine is responsible for the hospital care of a large part of the Galician population. There is heterogeneity in the Internal Medicine Services of our region that is not attributable only to the different levels of hospitals.

KEYWORDS: Internal Medicine. Survey. Care activity

No existen muchas publicaciones que recojan la actividad asistencial realizada por los internistas. En el año 2005 se publicó "El estudio socioprofesional de la Medicina Interna en España: Prospectiva 2010"3, elaborado por la Sociedad Española de Medicina Interna (SEMI) donde se analizaba la situación de la propia especialidad y su posible proyección futura. Para realizar dicho estudio se utilizaron 35 memorias de los SMI, que incluyeron tres gallegas, las del Hospital 
Juan Canalejo, el Complejo Hospitalario de Ourense (CHOU) y el Hospital Xeral Calde de Lugo, y 11 cuestionarios, uno de los cuales se realizó en el Hospital Juan Canalejo. Un año más tarde un original describe la actividad de los SMI del Sistema Público Andaluz 4 , y recientemente se publicó la "Encuesta SEMI sobre la oferta asistencial de los internistas en los hospitales del Sistema Público de Salud"1, donde se ofrece una visión panorámica sobre la organización asistencial (plantilla, hospitalización, consultas externas, interconsultas, investigación y docencia). Hasta el presente, en Galicia no se ha realizado ningún estudio similar.

La idea de realizar este estudio surgió como proyecto de la Sociedad Gallega de Medicina Interna. El objetivo era conocer la actividad asistencial de los internistas gallegos en cada centro hospitalario, su estructura, funcionamiento, carga de trabajo, etc., y así tener una foto real de la situación de nuestra especialidad en nuestra Comunidad Autónoma, y poner de manifiesto coincidencias y diferencias que existan entre los diferentes centros hospitalarios.

\section{Material y métodos}

La SOGAMI elaboró una encuesta, que fue presentada y aprobada por los miembros de la Junta Directiva 20092010, y que recoge diferentes aspectos de la actividad asistencial que cada Servicio de Medicina Interna llevó a cabo durante el año 2009. Fue cumplimentada por los representantes-vocales en la SOGAMI de cada hospital. En el caso de que no hubiera representante la Junta Directiva se puso en contacto con el SMI del centro encuestado y fue realizada por alguno de sus miembros.

El tipo de cuestionario permitía respuestas abiertas ya que sólo se formulaban las preguntas, sin establecer categorías de respuesta. La suma total de preguntas fue de 42. La estructura y contenido se dividió en ocho apartados que se citan a continuación: 1.- La organización del servicio, donde se incluía el número total de camas del centro hospitalario, número de camas a cargo de Medicina Interna, número aproximado de camas asignadas por adjunto, unidades dentro del servicio, internistas en otras áreas del hospital, estancia media pactada, estancia media realizada, número de pacientes/año. 2.- Personal, dentro del cual se preguntó por el número total de médicos de plantilla, jefes de servicio, jefes de sección, adjuntos y residentes. 3.- Organización de las guardias. A este respecto se averiguó el número de guardias por adjunto al mes, número de adjuntos y de residentes por guardia, número aproximado de ingresos y llamadas totales por guardia, otros especialistas médicos que realizan guardia de Medicina Interna, guardias específicas de especialidades, libranza de guardia, hora de comienzo, quién realiza los ingresos por la mañana y quién sigue a los pacientes pendientes de cama en el SMI.
4.- Consultas externas. Incluyó conocer cuántos internistas hacen exclusivamente consulta, número de consultas a la semana por adjunto, pacientes nuevos y revisiones por consulta, tiempo para ver un paciente nuevo y una revisión, tiempo dedicado a la consulta semanalmente, consultas monográficas y número de residentes en consultas. 5.- Interconsultas intrahospitalarias, donde se recogen el número de interconsultas a la semana, quién las realiza y si había interconsultores específicos por servicios. 6.- Universidad, el apartado donde se preguntaba si existe rotación organizada de alumnos de medicina. Los dos últimos apartados, 7.- Relación con Atención Primaria y 8.- Otras Actividades realizadas, eran preguntas con texto libre para describir la relación con Atención Primaria y reseñar otras actividades.

Para comparar algunas variables se clasificaron los hospitales en dos niveles: Hospitales Comarcales y Hospitales no Comarcales, incluyendo este último a los Hospitales de Área y a los Hospitales de Referencia. Los Hospitales Comarcales son: Hospital Da Costa, Hospital Virxe da Xunqueira, Hospital Comarcal de Monforte, Hospital Comarcal Da Barbanza, Hospital Comarcal Do Salnés, Hospital de Verín, y Hospital Comarcal de Valdeorras.

Se recogieron todos los datos y se incluyeron en una hoja de cálculo. Se realizó un estudio descriptivo de todas las variables mencionadas expresando las medidas de centralización y dispersión de las variables cuantitativas y el valor absoluto y porcentaje de las variables cualitativas.

\section{Resultados}

En total participaron y obtuvimos los datos de catorce de Ios dieciséis hospitales públicos de Galicia. No contestaron la encuesta el Hospital de Montecelo de Pontevedra y el Hospital Xeral de Vigo.

Los catorce hospitales encuestados suman un total de 6196 camas de las cuales el 22,1\% son responsabilidad de Medicina Interna. La Figura 1 muestra la distribución de camas por Hospital. El Complejo Hospitalario Universitario de A Coruña (CHUAC) es el que dispone de mayor número de camas, con un total de 1434, perteneciendo 362 (25\%) a Medicina Interna, mientras que el Hospital Virxe da Xunqueira es el de menor número total, 72 camas, de las que la mitad, 36, pertenecen a Medicina Interna.

Si se agrupan los hospitales por niveles y comparamos los no comarcales con los comarcales, objetivamos que en los primeros el 20,1\% de sus camas son de Ml, cifra que llega al 36,1\% en los comarcales. Hay 253 internistas contratados en los 14 Hospitales encuestados, de los cuales 28 trabajan en otras Áreas no dependientes de Medicina Interna. La media global es de 5,5 $\pm 3,1$ internistas por cada 100 camas de hospital; en los hospitales no comarcales es de $3,3 \pm 1,3$ y en los comarcales de $7,6 \pm 2,8$. 
Figura 1. Distribución de camas por hospitales. Representando camas a cargo de Ml y resto de camas de cada Centro Hospitalario. En la parte superior de cada columna se representa el tanto por ciento de camas a cargo de Ml en cada Centro

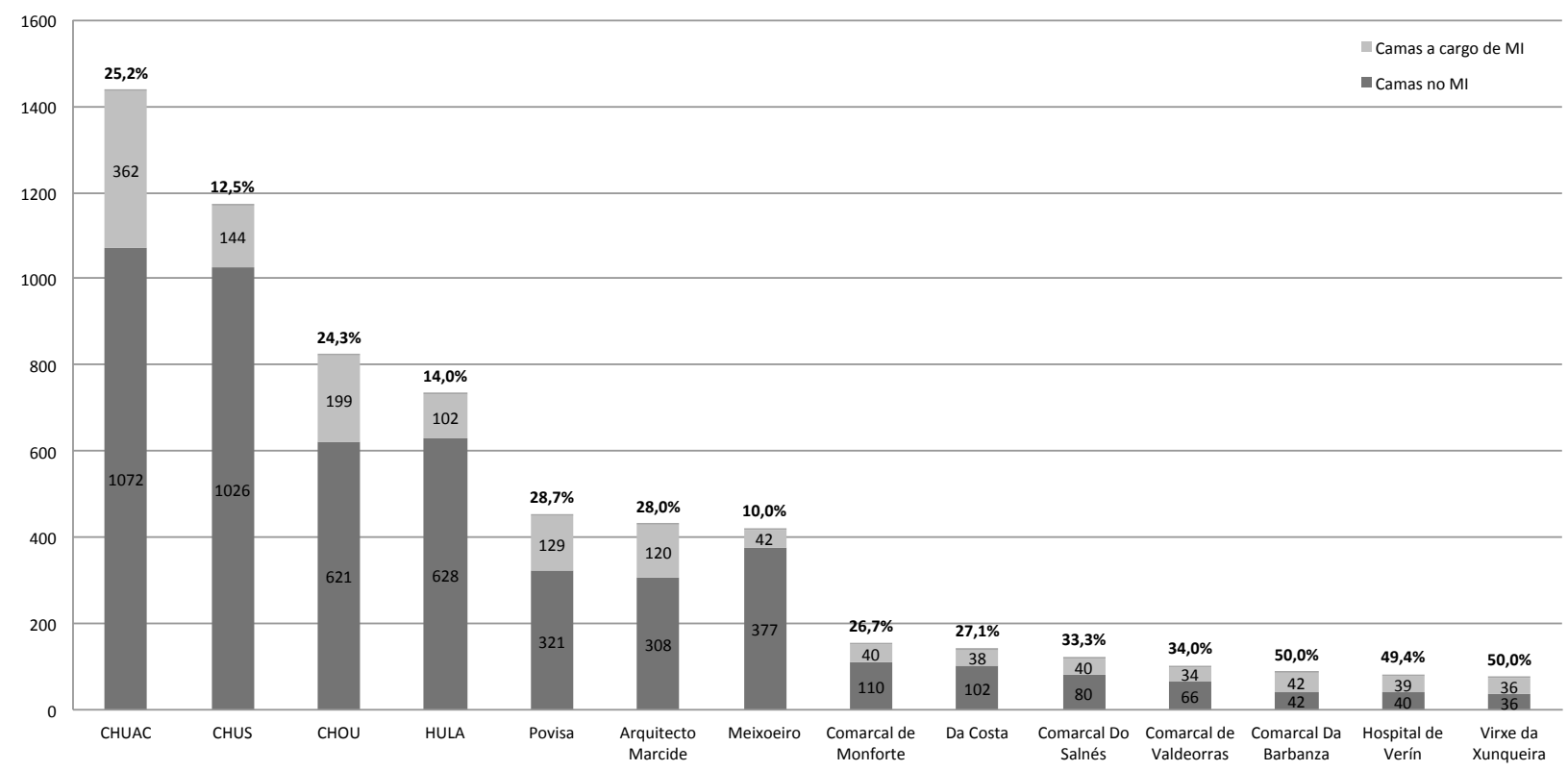

Tabla 1. Número de camas de Medicina Interna por médico de plantilla. Se muestran los datos declarados por cada Centro, y los datos calculados

\begin{tabular}{|l|c|c|c|c|c|c|c|c|c|c|c|c|c|c|}
\hline & CHUAC & CHUS & CHOU & HULA & Povisa & $\begin{array}{c}\text { Arquitecto } \\
\text { Marcide }\end{array}$ & Meixoeiro & $\begin{array}{c}\text { Comarcal } \\
\text { de } \\
\text { Monforte }\end{array}$ & Da Costa & $\begin{array}{c}\text { Comarcal } \\
\text { Do } \\
\text { Salnés }\end{array}$ & $\begin{array}{c}\text { Comarcal } \\
\text { de } \\
\text { Valdeorras }\end{array}$ & $\begin{array}{c}\text { Comarcal } \\
\text { Da } \\
\text { Barbanza }\end{array}$ & $\begin{array}{c}\text { Hospital } \\
\text { de Verín }\end{array}$ & $\begin{array}{c}\text { Virxe da } \\
\text { Xunqueira }\end{array}$ \\
\hline $\begin{array}{l}\text { Número de camas } \\
\text { por médico de } \\
\text { plantilla (datos } \\
\text { declarados) }\end{array}$ & 8,82 & 8,36 & 11,00 & 6,80 & 10,00 & 10,00 & 7,00 & 10,00 & 12,00 & 10,00 & 6,00 & 7,00 & 6,00 & 6,00 \\
\hline $\begin{array}{l}\text { Núm. calculado de } \\
\text { camas de Ml por } \\
\text { médico de plantilla } \\
\text { (salvo adj.sóloCons.) }\end{array}$ & 8,83 & 6,55 & 7,11 & 5,10 & 8,06 & 5,00 & 6,00 & 8,00 & 7,60 & 5,00 & 3,78 & 6,00 & 4,33 & 5,14 \\
\hline
\end{tabular}

La media de camas por cada médico de plantilla en Galicia según los datos declarados es de 8,5 \pm 2 , oscilando entre las 6 en los hospitales de Verín, Comarcal de Valdeorras y el de Virxe da Xunqueira y el máximo de 12 en el Hospital Da Costa. Si el cálculo se realiza teniendo en cuenta el número de camas declarado por cada Servicio y el número de facultativos, sin contar a los que sólo realizan consulta, la media es de 6,2 $\pm 1,6$. La Tabla 1 recoge estos datos por hospital, tanto los declarados como los calculados. Comparando los hospitales por niveles en los comarcales son $5,7 \pm 1,6$ camas calculadas por médico y $6,7 \pm 1,4$ en los demás.

En casi todos los centros hospitalarios el Servicio de Medicina Interna está formado por diferentes Unidades, excepto en los hospitales comarcales Do Salnés, Da Barbanza y de Virxe da Xunqueira, donde sólo existe Medicina Interna General. Las Unidades más prevalentes, además de Medicina Interna General en los 14 centros, son las de Crónicos y
Paliativos e Infecciosas en el 42,9\% y de Corta Estancia en el $35,7 \%$ de los centros. Sólo el hospital de Povisa tiene Unidad de Cuidados Intermedios perteneciente a Medicina Interna, y sólo en hospital Arquitecto Marcide, Hospitalización a Domicilio (HADO) está a cargo de Medicina Interna.

Hay 28 internistas que trabajan en otras áreas del hospital no dependientes de Ml, 8 de ellos en el Complejo Hospitalario Universitario de Santiago de Compostela (CHUS), 7 en el Hospital Lucus Augusti de Lugo (HULA) y 3 en el CHUAC. La distribución es la siguiente: 15 de ellos trabajan en el Servicio de Urgencias, 8 en HADO, 3 en la Unidad de Preingreso, 1 como oncólogo y 1 como endocrino. Cuatro hospitales no tienen internistas en otras áreas, son los de Arquitecto Marcide, Comarcal de Monforte, Da Costa y Comarcal Do Salnés.

La estancia media de hospitalización del Servicio de Medicina Interna en el año 2009 fue de 9,8 \pm 1,3 días mientras 
Figura 2. Estancia media de hospitalización del Servicio de Medicina Interna en el año 2009 por cada Hospital, y estancia media objetivo pactada para ese mismo año en ese mismo Hospital

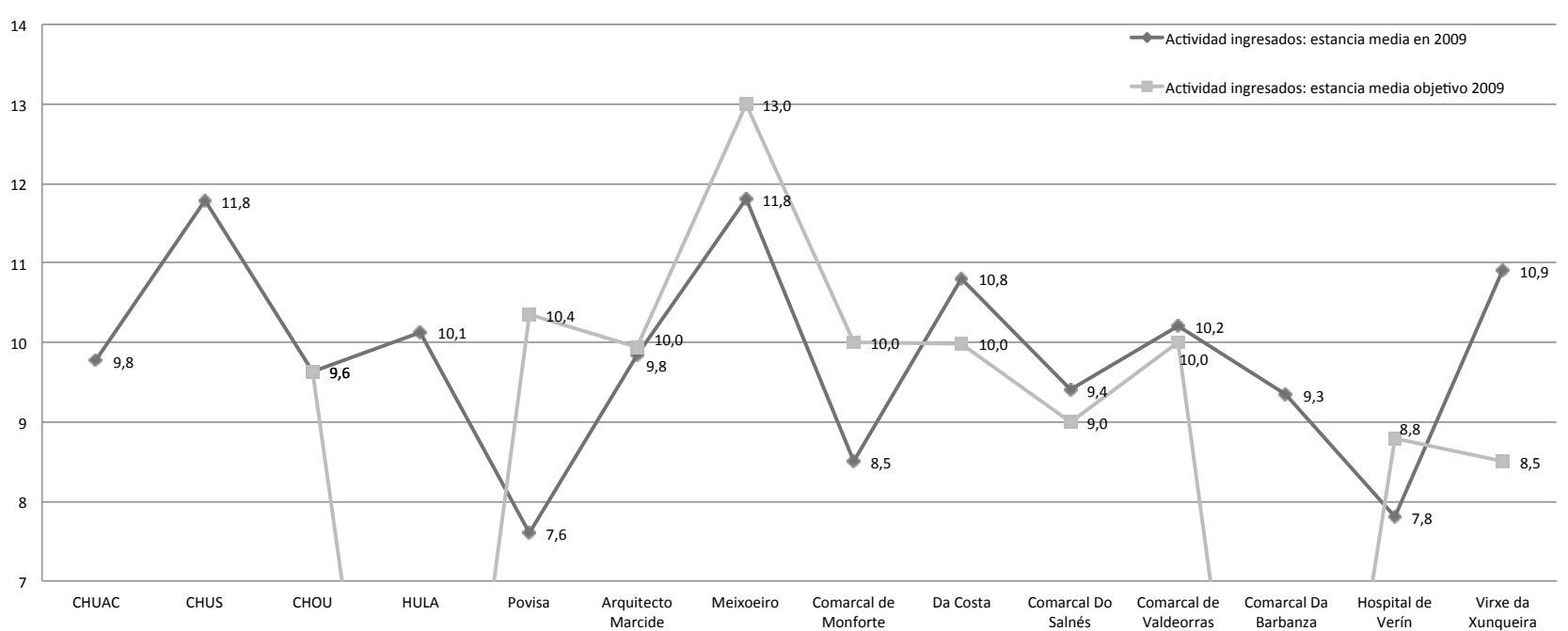

Figura 3. Actividad ingresados. № pacientes/año

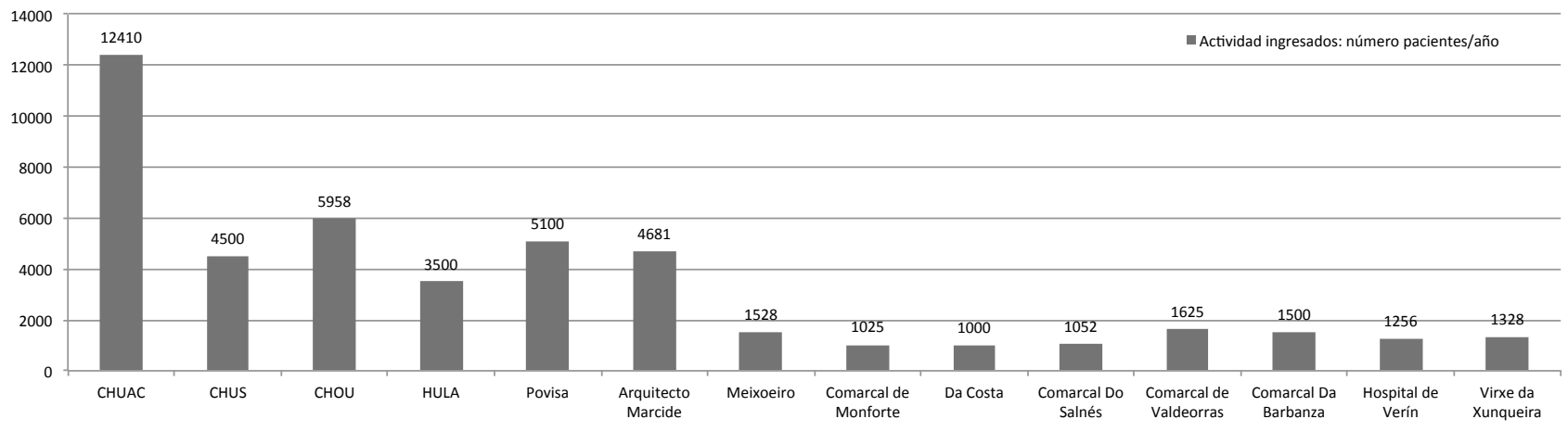

que la media de la estancia objetivo pactada ese mismo año fue de 9,9 $\pm 1,2$ días. En la Figura 2 se representa la media de la estancia de cada hospital y la media de la estancia objetivo pactada, si bien este último dato no se pudo obtener en todos los centros. La estancia en los comarcales es algo menor que en los demás: 9,6 $\pm 1,2$ frente a 10,1 $\pm 1,4$ días.

Con respecto a la actividad de pacientes ingresados en el año 2009 fueron atendidos 46.463 pacientes en los Servicios de Medicina Interna de los 14 centros, con la distribución mostrada en la Figura 3. El 81,1\% ingresaron en hospitales no comarcales.

Con los datos aportados por la encuesta se pudo calcular la media de pacientes ingresados en Medicina Interna al año por cada médico de plantilla en Galicia, que fue de 194,6 $\pm 55,1$, y la media en cada hospital (Figura 4). Si compara- mos los hospitales, dicha media en los comarcales fue de $171,9 \pm 33,7$ frente a 217,2 \pm 65,2 en los demás.

Respecto al apartado de Personal la Tabla 2 resume el número de residentes, médicos adjuntos, jefes de sección y de servicio en cada uno de los hospitales públicos gallegos. Indicando también la media de cada uno de ellos en Hospitales Comarcales y no Comarcales.

La media de guardias de MI por adjunto en Galicia es de $3,5 \pm 0,9$ al mes con un mínimo de 2 en el CHUAC, y un máximo de 5,5 en el Hospital Virxe da Xunqueira. Sólo en 2 de los hospitales, CHUAC y Meixoeiro, no se libran las guardias. La hora de comienzo de la guardia en día laborable suele ser a las 15 horas a excepción de los hospitales de Povisa, Meixoeiro, Comarcal Do Salnés, Comarcal de Valdeorras y Verín que comienzan entre 8 y 9 horas. La carga de trabajo en la guardia varía de unos centros a otros: la media de camas por personal de guardia, incluido resi- 
Tabla 2. Distribución del personal por hospitales, y medias agrupando por tipo de hospital. En el grupo superior están los Hospitales no Comarcales, y en el grupo inferior los Hospitales Comarcales

\begin{tabular}{|c|c|c|c|c|c|c|c|c|}
\hline & MEDIA & CHUAC & CHUS & $\mathrm{CHOU}$ & HULA & Povisa & $\begin{array}{l}\text { Arquitecto } \\
\text { Marcide }\end{array}$ & Meixoeiro \\
\hline Jefes de servicio & 1,1 & 1 & 2 & 1 & 1 & 1 & 1 & 1 \\
\hline Jefes de sección & 4,0 & 7 & 6 & 5 & 3 & 2 & 4 & 1 \\
\hline Adjuntos & 19,6 & 34 & 21 & 26 & 18 & 13 & 20 & 5 \\
\hline $\mathrm{N}^{0}$ total de residentes & 6,1 & 10 & 9 & 6 & 4 & 4 & 5 & 5 \\
\hline
\end{tabular}

\begin{tabular}{|l|c|c|c|c|c|c|c|}
\hline & MEDIA & $\begin{array}{c}\text { Comarcal de } \\
\text { Monforte }\end{array}$ & Da Costa & $\begin{array}{c}\text { Comarcal Do } \\
\text { Salnés }\end{array}$ & $\begin{array}{c}\text { Comarcal de } \\
\text { Valdeorras }\end{array}$ & $\begin{array}{c}\text { Comarcal Da } \\
\text { Barbanza }\end{array}$ & $\begin{array}{c}\text { Hospital de Verín } \\
\text { Xunqueira }\end{array}$ \\
\hline Jefes de servicio & 0,9 & 1 & 1 & 1 & 1 & 0 & 1 \\
\hline Jefes de sección & 0,1 & 0 & 0 & 0 & 1 & 0 & 0 \\
\hline Adjuntos & 6,4 & 4 & 6 & 7 & 7 & 7 & 0 \\
\hline$N^{\circ}$ total de residentes & 0,0 & 0 & 0 & 0 & 0 & 0 & 0 \\
\hline
\end{tabular}

Tabla 3. Distribución de pacientes nuevos al año en consultas externas por hospitales

\begin{tabular}{|l|c|c|c|c|c|c|c|c|c|c|c|c|c|}
\hline & TOTAL & CHUAC & CHUS & CHOU & HULA & Povisa & $\begin{array}{c}\text { Arquitecto } \\
\text { Marcide }\end{array}$ & Meixoeiro & $\begin{array}{c}\text { Comarcal } \\
\text { de } \\
\text { Monforte }\end{array}$ & Da Costa & $\begin{array}{c}\text { Comarcal Do } \\
\text { Salnés }\end{array}$ & $\begin{array}{c}\text { Comarcal } \\
\text { de } \\
\text { Valdeorras }\end{array}$ & $\begin{array}{c}\text { Comarcal } \\
\text { Da } \\
\text { Barbanza }\end{array}$ \\
$\begin{array}{l}\text { Hospital } \\
\text { de Verín }\end{array}$ & $\begin{array}{c}\text { Virxe da } \\
\text { Xunqueira }\end{array}$ \\
\hline $\begin{array}{l}\text { Pacientes } \\
\text { al año }\end{array}$ & 42991 & 4906 & 3024 & & 7648 & 3337 & 6844 & 2920 & 1043 & -- & 1669 & 5631 & 730 \\
\hline
\end{tabular}

Figura 4. Media de pacientes ingresados al año por médico de plantilla.

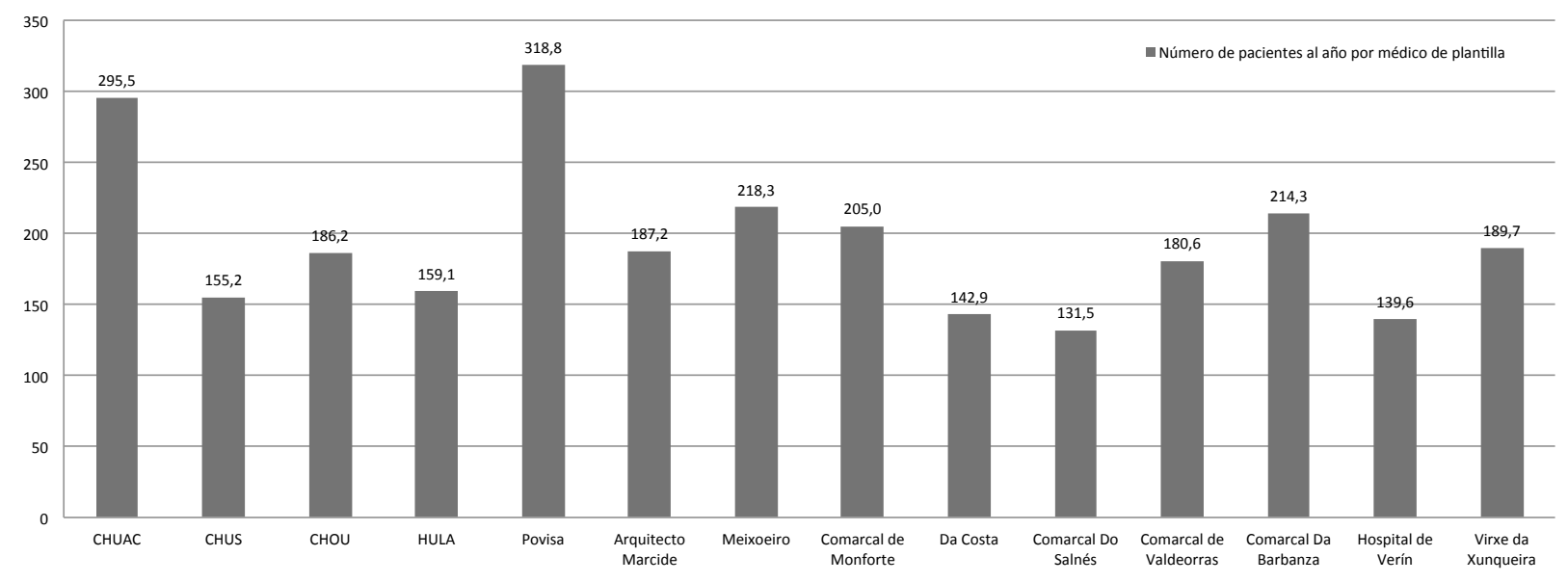

dentes de guardia, es 34,5 $\pm 11,3$, siendo el mínimo de 14 camas en el Meixoeiro y el máximo de 55,3 en Povisa, la media de llamadas al busca es de 11,5 $\pm 6,9$ por personal de guardia con un mínimo de 2,8 en el CHUS y un máximo de 22,5 en el Hospital Virxe da Xunqueira y la media de ingresos es de 5,4 $\pm 1,4$ por personal de guardia siendo el mínimo de 2,8 en el Hospital Arquitecto Marcide y el máximo de 7,5 en el Hospital Comarcal Do Salnés.
La valoración de ingresos por la mañana no es homogénea en los distintos hospitales; en el 35,7\% lo realiza el personal de guardia de $\mathrm{Ml}$; en el 28,6\% se hace cargo el SMl, en el $14,3 \%$ el Servicio de Urgencias, también en un 14,3\% un busca llevado por internistas asignado para ese cometido $y$ en el 7,1\% nadie. En el CHUS a veces lo realiza la UMAP (Unidad Médica de Alta Precoz). La valoración de pacientes ingresados pendientes de cama, la realiza en el $21,4 \%$ de Ios casos la guardia, en el $28,6 \%$ el SMl, en el $21,4 \%$ el 


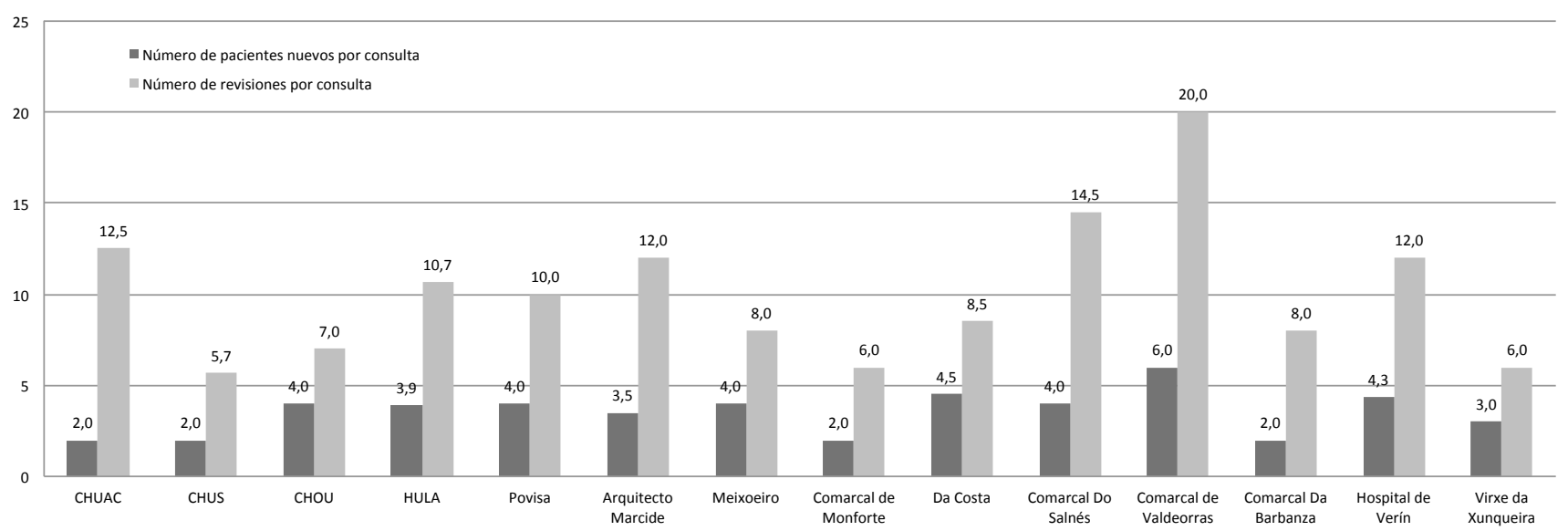

Tabla 4. Distribución de consultas monograficas por hospitales

\begin{tabular}{|l|c|c|c|c|c|c|c|c|}
\hline & CHUAC & CHUS & CHOU & HULA & Povisa & Arquitecto Marcide & Meixoeiro & Comarcal de Monforte \\
\hline Infecciosas/SIDA & $\mathrm{x}$ & $\mathrm{x}$ & $\mathrm{x}$ & & $\mathrm{x}$ & & & \\
\hline ETEV & & & & $\mathrm{x}$ & & $\mathrm{x}$ & $\mathrm{x}$ & \\
\hline Factores de riesgo cardiovascular & & & $\mathrm{x}$ & $\mathrm{x}$ & & & $\mathrm{x}$ & \\
\hline Pluripatología & & & $\mathrm{x}$ & $\mathrm{x}$ & & & & \\
\hline Hepatología & $\mathrm{x}$ & $\mathrm{x}$ & & & & & & \\
\hline HTA & & & & & & & & \\
\hline Lípidos & & & & & & & & \\
\hline Insuficiencia cardiaca & & & & $\mathrm{x}$ & & & & \\
\hline Patologia Crónica & & & & & & & & \\
\hline Alta resolución & & & & & & & & $x$ \\
\hline
\end{tabular}

Servicio de Urgencias; en los hospitales de Povisa y Virxe da Xunqueira no existen pacientes pendientes de cama, en el Hospital Comarcal Do Salnés se hace responsable el que lo ingresa y en el CHUS a veces la UMAP según disponibilidad. En las guardias de Medicina Interna participan otras 11 especialidades médicas, siendo los neumólogos, endocrinos, neurólogos y reumatólogos las especialidades más frecuentes.

Para calcular el trabajo realizado en Consultas Externas no disponemos de datos completos del CHOU ni del Hospital Da Costa. Considerando los demás, el número de pacientes nuevos al año vistos en consultas externas es de 42.991, y su distribución por hospitales se refleja en la Tabla 3. El $7,6 \%$ de los internistas sólo trabajan en Consultas Externas. La Figura 5 recoge el número de pacientes nuevos y revisiones que atiende cada internista en cada consulta por hospitales.

Los residentes tienen consultas de Medicina Interna solamente en el 42,9\% de los hospitales. La mayoría de los hospitales, 10 de los 14, tienen consultas post-hospitalización, mientras que monográficas hay en 8 centros, siendo la más prevalente la de enfermedades infecionas/VIH. A excepción del Hospital Comarcal de Monforte donde existen consultas de alta resolución y de patología crónica, en el resto de hospitales comarcales no existen consultas monográficas (Tabla 4). El tiempo disponible para atender a un paciente nuevo es de 20 ó 30 minutos, con un mínimo de 15 en el CHUS y máximo de 45 en el Arquitecto Marcide, y para las revisiones oscilan entre los 10 y 15 minutos. 
Tabla 5. Relación con Atención Primaria

\begin{tabular}{|c|c|c|c|c|c|c|c|c|c|c|c|c|c|c|}
\hline & CHUAC & CHUS & $\mathrm{CHOU}$ & HULA & Povisa & $\begin{array}{l}\text { Arquitecto } \\
\text { Marcide }\end{array}$ & Meixoeiro & $\begin{array}{l}\text { Comarcal } \\
\text { de } \\
\text { Monforte }\end{array}$ & Da Costa & $\begin{array}{c}\text { Comarcal } \\
\text { Do } \\
\text { Salnés }\end{array}$ & $\begin{array}{l}\text { Comarcal } \\
\text { de } \\
\text { Valdeorras }\end{array}$ & $\begin{array}{c}\text { Comarcal } \\
\text { Da } \\
\text { Barbanza }\end{array}$ & $\begin{array}{l}\text { Hospital } \\
\text { de Verín }\end{array}$ & $\begin{array}{c}\text { Virxe da } \\
\text { Xunqueira }\end{array}$ \\
\hline Relación con primaria & NO & NO & SI & SI & SI & $\mathrm{SI}$ & SI & NO & SI & SI & SI & NO & SI & SI \\
\hline $\begin{array}{l}\text { Asistencia Centro de } \\
\text { Salud }\end{array}$ & NO & NO & SI & SI & NO & SI & NO & NO & NO & NO & SI & NO & NO & NO \\
\hline Teléfono & NO & NO & SI & SI & NO & SI & NO & NO & SI & SI & SI & NO & $\mathrm{SI}$ & NO \\
\hline Formación & NO & NO & SI & SI & SI & NO & SI & NO & SI & NO & NO & NO & SI & SI \\
\hline
\end{tabular}

Figura 6.Distribución por hospitales de las interconsultas a la semana

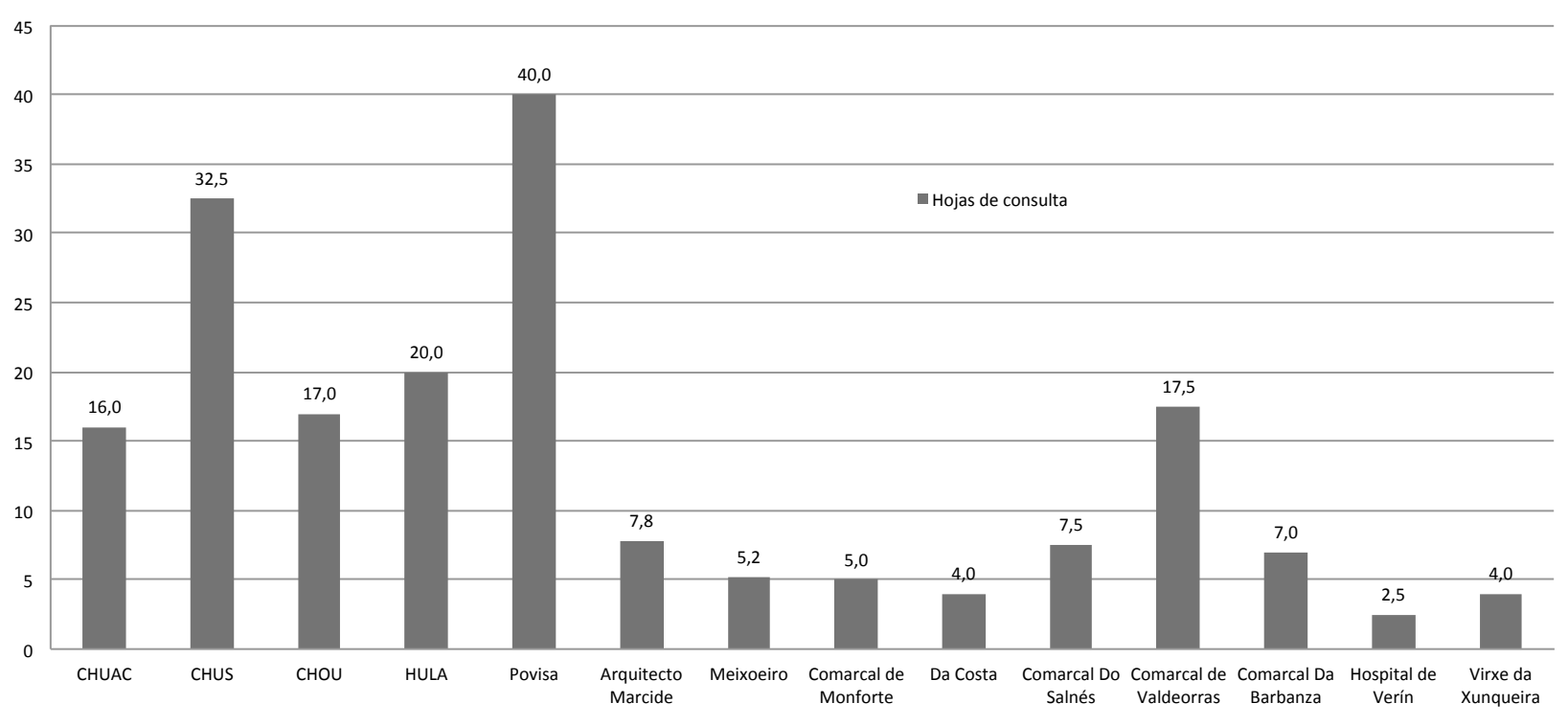

Las interconsultas hospitalarias son realizadas por la guardia de Medicina Interna en 3 de los centros, por un staff a tiempo parcial en Povisa y repartidas entre los médicos del servicio en la mayoría de los hospitales. La carga de trabajo que supone es una media de 13,3 $\pm 11,4$ interconsultas por Hospital a la semana (Figura 6).

En todos los Hospitales hay rotaciones de alumnos universitarios, pero en 6 de ellos son sólo en verano: Meixoeiro, Comarcal de Valdeorras, Comarcal Da Barbanza, Verín, Virxe da Xunqueira y Da Costa.

La relación con Atención Primaria es heterogénea y centrada en tres apartados: asistencia al Centro de Salud, consulta telefónica o reuniones de formación. La Tabla 5 recoge la distribución de dichos parámetros por hospital.

Otras actividades llevadas a cabo desde los SMI son variadas: el HULA tiene una Unidad de Insuficiencia Cardíaca con contacto telefónico con Atención Primaria; en el Hospital Comarcal de Monforte el registro Holter es labor de los internistas; en el Hospital Da Costa un internista realiza las endoscopias digestivas y en el Hospital Comarcal Da Bar- banza los internistas de manera ocasional hacen guardia de hemodiálisis.

\section{Discusión}

La Medicina Interna en España, por sus características más generalistas, es una de las especialidades con mayor implantación en los hospitales ${ }^{3}$. Según datos publicados en 2005 en el área INSALUD era de 2,5 internistas/100 camas y 4/100 en los comarcales ${ }^{3}$. En los hospitales del SERGAS la relación actual es superior, con un ratio de 5,5 internistas / 100 camas y en los comarcales de 7,6 internistas / 100 camas. El número de camas de Medicina Interna respeto al número total de cada hospital es variable dependiendo del tamaño del centro hospitalario, su ubicación y la especialización de dicho centro ${ }^{3}$, y además tienen un gran margen de variabilidad debido a la política local de gestión de recursos del propio centro y a la organización del mismo como hemos comprobado con los resultados del presente estudio. Según el estudio Socioprofesional de la Medicina Interna en España ${ }^{3}$, en los hospitales comarcales el número de camas a cargo de Medicina Interna no debe ser inferior 
al 33\%, en los hospitales medios al 15\% y en los de alta especialización al 10\%. En Galicia los Servicios de Medicina Interna de los hospitales públicos cumplen esta recomendación, gestionando de media el 20\% de las camas de los centros de área y referencia y el $36 \%$ de las camas de los hospitales comarcales.

Los internistas gallegos atienden a uno de cada cuatro 0 cinco pacientes hospitalizados, cifras similares a las publicadas recientemente sobre los hospitales nacionales del sistema público de salud ${ }^{1}$ y algo mayores a las publicadas previamente ${ }^{2}$. Cada médico internista es responsable de 195 pacientes ingresados cada año. Estos datos confirman la manifiesta importancia de la especialidad en la atención hospitalaria de nuestra Comunidad Autónoma.

El internista posee un papel clásico como generalista hospitalario por su visión holística de los pacientes, capacidad integradora y mayor eficiencia a la hora de tomar decisiones diagnósticas y terapéuticas, que lo hacen imprescindible en los hospitales comarcales y fundamental en los hospitales de área y referencia. Pero además cuenta con un plus al ejercer de "superespecialista" en patologías 0 áreas que carecen de titulación específica 0 formación reglada como puede ser hipertensión, riesgo vascular, crónicos, cuidados paliativos, infecciosas y hospitalización a domicilio entre otras muchas; este papel se vuelve también imprescindible en los hospitales de referencia y en muchos hospitales de área. La superespecialización, a la que se debe llegar a través de las Áreas de Capacitación Específica, debe ser un valor añadido a nuestro trabajo sin perder la condición intrínseca de internistas, que es la del médico que atiende globalmente al paciente. En el SERGAS las secciones 0 unidades que se incluyen en los SMI son múltiples y variadas, dependiendo de la complejidad del centro y de la política de salud desarrollada, lo que coincide con lo reflejado en otros estudios ${ }^{3}$. En nuestra Comunidad Autónoma, once de los catorce hospitales tiene alternativas a la hospitalización convencional, superando la realidad del resto de hospitales nacionales del Sistema Público de Salud ${ }^{1}$. La más frecuente es la de Unidad de Cuidados Paliativos que está presente en casi la mitad de los centros. También en el resto de los hospitales de España ${ }^{1}$ estas unidades son las más frecuentes. Por otro lado, la hospitalización domiciliaria es una unidad específica en el $34 \%$ de los hospitales del Sistema Público de Salud ${ }^{1}$ pero sólo el 30\% dependen de Servicios de Medicina Interna. Nuestro estudio confirma que en los centros del SERGAS sólo el 7\% dependen de Ml. Otra alternativa a la hospitalización convencional, las Unidades de Corta Estancia, están implantadas en algo más de la tercera parte de nuestros hospitales.
La media de la estancia global fue de 9,8 días, algo menor que la publicada en otros estudios nacionales, que resultó de 10 días $^{2,3}$ y 11,8 días 4 ; la obtenida por los hospitales comarcales fue ligeramente menor, 9,6 días, coincidiendo con lo recogido en otros trabajos ${ }^{2,4}$; este hecho probablemente ocurra, al menos en parte, porque estos hospitales atiendan a pacientes con patologías con mejor pronóstico, y aquellos más graves y complejos sean remitidos a hospitales de área 0 de referencia. Si comparamos nuestros datos con los nacionales la estancia de nuestros hospitales comarcales es mayor, superando ligeramente los 8,9 días de su media².

La atención continuada está organizada en todos los hospitales mediante la realización de guardias de presencia física. La media de 3,5 guardias mensuales es similar a la recogida por otros estudios nacionales ${ }^{1}$. La carga de trabajo que se soporta en ellas es muy variable dependiendo de las características concretas de cada hospital.

La actividad ambulatoria ha sido más difícil de evaluar al existir algunas respuestas menos estructuradas, en ocasiones amplias y en algunos casos poco concretas. La consulta monográfica más habitual es la consulta de enfermedades infecciosas incluida la infección por VIH, lo que coincide con otros estudios ${ }^{1}$. Sólo uno de nuestros hospitales tiene consulta de alta resolución; este tipo de consultas parecen esenciales para disminuir los ingresos inadecuados y para agilizar el diagnóstico de patologías graves y así mejorar la eficiencia de nuestro trabajo. Respecto al tiempo asignado en consultas externas, los tiempos medios asignados para un paciente nuevo, de 30 minutos, y para una revisión de 15 minutos, son los que se consideran adecuados ${ }^{1}$.

Entre las funciones asistenciales de especialista en Medicina Interna figura la de dar respuesta a las consultas que se le hacen desde otros servicios fundamentalmente quirúrgicos, médico-quirúrgicos y psiquiátricos ${ }^{5}$. La resolución de estas interconsultas conlleva una sobrecarga asistencial poco estudiada ${ }^{6,7}$. Es necesario recoger esta información de manera estandarizada puesto que supone una parte importante y progresivamente mayor del trabajo que realizamos los internistas. Conocer con precisión estos datos ayudaría a determinar cuál es la organización más eficiente. En el $71,4 \%$ de los Servicios de Medicina Interna gallegos esta tarea se reparte entre los distintos médicos del servicio; ningún servicio tiene facultativo asignado en jornada completa y sólo un centro tiene un staff a tiempo parcial. La diferencia es evidente con otros hospitales nacionales, donde el $25 \%$ tienen adjunto a tiempo completo y el $42 \%$ a tiempo parcial ${ }^{1}$.

Respecto a la relación con Atención Primaria, 4 de los 14 hospitales no tienen ninguna relación con el primer escalón 
sanitario; y de los 10 que sí la tienen, un tercio de ellos es sólo una relación formativa con participación conjunta en cursos de formación. La colaboración con Primaria, especialmente en el ámbito asistencial debería estar más extendida porque mejora la eficacia y la eficiencia de la atención de los pacientes. Sin embargo los dos niveles parece que se desconocen e históricamente mantienen un distanciamiento en la comunicación y coordinación profesional y asistencial, que hace que la fragmentación de la asistencia sanitaria se convierta en uno de los mayores problemas del Sistema sanitario. ${ }^{5}$

La principal limitación de este estudio deriva de la propia naturaleza del método de obtención de los datos, una encuesta, cuyos resultados pueden obedecer a interpretaciones diferentes de las cuestiones presentadas, y existir una importante carga de subjetividad y sinceridad en las respuestas ${ }^{1}$. A pesar de ello, su valor estriba en ser el primer trabajo que se realiza en Galicia que analiza y da a conocer diferentes aspectos de la organización asistencial de los Servicios de Medicina Interna de los hospitales públicos. Además la participación en esta encuesta ha sido elevada ya que disponemos de los datos de 14 de los 16 hospitales del SERGAS.

Los resultados de este trabajo confirman la gran relevancia de los Servicios de Medicina Interna en la atención sanitaria hospitalaria de la población gallega, con responsabilidad directa en el cuidado de pacientes mayor que el observado en la media de los hospitales nacionales. Este dato debe servir para transmitir la importancia de nuestra especialidad a los gestores sanitarios y a otros especialistas médicos.

Otro aspecto muy significativo de este trabajo ha sido conocer las coincidencias y diferencias que existen en el funcionamiento de los distintos Servicios de Medicina Interna, que no guardan relación sólo con el tamaño del hospital, y constatar que existe una gran heterogeneidad tanto en la organización como en el funcionamiento de los mismos. La historia de cada hospital y el desarrollo de cada uno de ellos son factores que explican, al menos en parte, estas diferencias. Además, la propia naturaleza del médico internista, con una sólida formación general, que le permite adaptarse a las distintas circunstancias y necesidades locales de atención sanitaria de la población y adaptarse también a los cambios que ocurren a lo largo del tiempo, contribuyen también a la diversidad del internismo gallego.

El conocer estos hechos nos permite saber cómo realizamos nuestro trabajo, compararnos, detectar defectos en nuestra organización o método, encontrar oportunidades de mejora, y modificar y mejorar nuestra práctica clínica diaria para seguir avanzando en el mejor cuidado de nuestros pacientes.

\section{Bibliografía}

1. Losa, J.E, Zapatero, A, Barba, R, Marco J, Plaza S, Canora J. Encuesta SEMI sobre la oferta asistencial de los internistas en los hospitales del Sistema Público de Salud. Rev Clin Esp. 2011; 211(5): 223-32.

2. Barba Martín R, Marco Martínez J, Emilio Losa J, Canora Lebrato J, Plaza Canteli S, Zapatero Gaviria A. Análisis de 2 años de actividad de Medicina Interna en los hospitales del Sistema Nacional de Salud. Rev Clin Esp.2009;209(10):459-66.

3. Alfredo del Campo Martín. Sociedad Española de Medicina Interna. Estudio Socioprofesional de la Medicina Interna en España: Prospectiva 2010. Dirección del estudio: Medical Science Service. 2005. p. 21-22.

4. De San Román y de Terán CM, Guijarro Merino R. Servicios hospitalarios de Medicina Interna. Una descripción de la actividad en el Sistema Público de Andalucía en 2002. Rev Clin Esp. 2006; 206 (1): 4-11.

5. La Medicina Interna como modelo de práctica clínica. Sociedad Gallega de Medicina Interna. 2009. p. 179 ISBN: 9788461261000

6. García Lázaro M, Lucena Merino M.M, Montero Pérez-Barquero M, Sanchez Guijo P. Estudio de la actividad asistencial de Medicina Interna en relación a las interconsulta An. Med. Interna (Madrid). 2003; 20 (10): 510-514.

7. Monte Secades R, Rabuñal Rey R, Rigueiro Veloso MT, García Pais MJ, Casariego Vales E, Guerrero Lombardía J. Papel del internista como consultor de servicios quirúrgicos. Rev Clin Esp. 2004; 204 (7): 345-50. 